\begin{tabular}{|c|l|}
\hline Title & Photochemical fabrication of molecular devices \\
\hline Author(s) & Naito, Toshio; Sugawara, Hidey uki; Inabe, Tamotsu; Miyamoto, Takeshi; Niimi, Hironobu; A sakura, Kiyotaka \\
\hline Citation & $\begin{array}{l}\text { Journal of Non-Crystalline Solids, 352(23.25), 2628 } \\
\text { https://doi.org/10.1016/.jnoncrysol.2006.01.097 }\end{array}$ \\
\hline Issue Date & $2006-07-15$ \\
\hline Doc URL & http://hdl.handle.net/2115/14564 \\
\hline Type & article (author version) \\
\hline File Information & JON-SC.pdf \\
\hline
\end{tabular}

Instructions for use 
Elsevier Editorial System(tm) for Journal of Non-Crystalline Solids

Manuscript Draft

Manuscript Number:

Title: Photochemical fabrication of molecular devices

Article Type: Conference Paper

Section/Category: AIOM 2005 Conference

Keywords: Conductivity C260; Doping D230; Photoinduced Effects P210; Photosensitivity P242

Corresponding Author: $\mathrm{Dr}$ Toshio Naito, $\mathrm{PhD}$

Corresponding Author's Institution: Hokkaido University

First Author: Toshio Naito, $\mathrm{PhD}$

Order of Authors: Toshio Naito, PhD; Hideyuki Sugawara, MD; Tamotsu Inabe, PhD; Takeshi Miyamoto, MD; Hironobu Niimi, PhD; Kiyotaka Asakura, PhD

Manuscript Region of Origin:

Abstract: We present a novel way to fabricate electronic devices from a molecular charge-transfer salt $\mathrm{Ag}(\mathrm{DM}) 2$ by simple illumination, which realizes a junction-structure in the single molecular crystal. The electrical conductivity of the selected parts of samples can be controlled by illimination time and power. The electrical behavior gradually turned semiconducting from metallic one by illumination. X-ray photoelectron spectroscopy, Raman spectroscopy and X-ray absorption fine structure indicated that illumination should transfer some electrons from the conducting DM columns to the Ag ions. The X-ray powder diffraction pattern indicated that original crystal lattice was maintained after the illumination. These observations imply that the illumination practically effected doping (or rather dedoping) on the material.

PACS: $73.40 . \mathrm{Ei} ; 73.40 . \mathrm{Ns}$ 


\title{
Photochemical fabrication of molecular devices
}

\author{
Toshio Naito a,b,*, Hideyuki Sugawara ${ }^{\mathrm{b}}$, Tamotsu Inabe ${ }^{\mathrm{b}}$, Takeshi Miyamoto ${ }^{\mathrm{c}}$, \\ Hironobu Niimi ${ }^{\text {c,d }}$, Kiyotaka Asakura ${ }^{\text {c }}$ \\ ${ }^{a}$ Creative Research Initiative "Sousei” (CRIS), Hokkaido University,Kita 21, Nishi 10, Kita-ku, Sapporo, \\ Hokkaido 001-0021, Japan \\ ${ }^{\mathrm{b}}$ Division of Chemistry, Graduate School of Scinece, Hokkaido University, Kita 10, Nishi 8, Kita-ku, \\ Sapporo, Hokkaido 060-0810, Japan \\ ${ }^{\mathrm{c}}$ Catalysis Reasearch Center (CRC), Hokkaido University, Kita 21, Nishi 10, Kita-ku, Sapporo, Hokkaido \\ 001-0021, Japan \\ ${ }^{d}$ CREST-JST
}

Received $* * * * * * *$; received in revised form $* * * * 2005$

\begin{abstract}
We present a novel way to fabricate electronic devices from a molecular charge-transfer salt $\operatorname{Ag}(\mathrm{DM})_{2}$ by simple illumination, which realizes a junction-structure in the single molecular crystal. The electrical conductivity of the selected parts of samples can be controlled by illimination time and power. The electrical behavior gradually turned semiconducting from metallic one by illumination. X-ray photoelectron spectroscopy, Raman spectroscopy and $\mathrm{X}$-ray absorption fine structure indicated that illumination should transfer some electrons from the conducting DM columns to the Ag ions. The X-ray powder diffraction pattern indicated that original crystal lattice was maintained after the illumination. These observations imply that the illumination practically effected doping (or rather dedoping) on the material.
\end{abstract}

PACS: $\quad$ 73.40.Ei; 73.40.Ns 


\section{Introduction}

A large number of molecular conductors have been reported to date [1-5]. Most of them contain planar $\pi$-conjugated molecules with multi-redox reactivities, which exist as metastable partially charged radical ions in the conducting solids. In general, their electrical conduction is supported by the unpaired electrons delocalized through $\pi$-bands, i.e. overlaps of the $\pi$-molecular orbitals among adjacent molecular radical-ions. They usually have structurally unique and various conduction pathways such as columns, sheets, ladders and spirals, depending on the sturctures and arrangements of constituent molecular species. Some of them also contain photosensitive chemical species such as silver ions and bypyridyl derivatives, which are not involved in the conduction pathways. Such solids are generally stable in ambient conditions. However, they could be reactive under illumination of ultraviolet and visible (UV-VIS) light, where electron transfer could occur between the photosensitive and the molecular species responsible for the conduction. As a result, illumination could irreversibly increase or decrease the amount of conduction electrons by their addition or removal. In combination with photolithography or light-focusing technique, this might open a new way to control irreversibly electrical properties of a particular part of a molecular solid, which would lead to fabrication of devices with junction-strutures. In this paper, we present an optical control method of electrical properties of molecular solids together with its mechanism. 


\section{Experimental}

The silver salt of dimethyl-dicyanoquinonediimine $(\mathrm{DM}), \operatorname{Ag}(\mathrm{DM})_{2}$, was prepared according to Ref. [6]. The electrical resistivity measurements were carried out using a standard 4-probe method with gold wires and gold paste as electrical contacts. A part of the sample (a single crystal or a pressed pellet) or the whole sample was continuously illuminated with UV-VIS light (200-1100 nm) using an optical fiber. Subsequently, the physical measurements were carried out for the sample using conventional procedures in the dark. Parallel measurements of the pristine samples were also conducted for checking out any artefacts. After the measurements, the illumination was recommenced on the same sample and further illumination effects were investigated. The details of illuminations and measurements of physical and spectroscopic properties were described in earlier papers [7-9].

\section{Results and discussion}

DM forms charge tran sfer salts with various kinds of metals. Owing to its moderate electron accepting ability, the DM molecules usually exist as radical anions with fractional valency in these salts. In the crystal of $\mathrm{Ag}(\mathrm{DM})_{2}$, the planar radical anions of DM stack to form a column ar structure (Fig. 1). The unpaired electrons on the radical anions delocalize along these columns, 
resulting in metallic conduction. All the DM anions are coordinated to the Ag ions through the $=\mathrm{N}-\mathrm{CN}$ groups at both ends of the fully $\pi$-conjugation system on the molecule. It was observed that $\mathrm{Ag}(\mathrm{DM})_{2}$ qualitatively altered its electrical properties after illumination with wavelengths of 200-1100 nm: the temperature-dependence of a partially illuminated single crystal of $\mathrm{Ag}(\mathrm{DM})_{2}$ was qualitatively explained as a sum of the electrical behavior of the illuminated and the non-illuminated parts of the sample (Fig. 2) [8]. In the range of 200-1100 nm, wavelength-dependence was not observed. The duration of illumination required for the change of physical properties varied from a few minutes to a few weeks depending mainly on the power of the light and the temperature.

Firstly, let us discuss what is happening in the sample during illumination. In the previous paper [8] we reported that X-ray photoelectron spectroscopy (XPS) was powerful tool to determine the chemical states of the illuminated part of the sample as well as how clear the borderline between the illuminated and the pristine parts of the sample was. The high-resolution XPS demonstrated that bulk silver $(\operatorname{Ag}(0))$ was detected exclusively in the illuminated area while silver(I) $(\operatorname{Ag}(\mathrm{I}))$ ions remained unaffected in the non-illuminated area. In addition, the borderline was so sharp and clear that $\operatorname{Ag}(0)$ was not detected even in adjacent parts of the illuminated area. If the charge (electron) transfer actually occurred between the Ag and the DM ions, the XPS of the atoms in the DM ions should be consistent with the XPS of silver atoms. In fact, the XPS of nitrogen atoms in the illuminated sample clearly indicated that the DM radical 
an ions were oxidized toward neu tral DM molecules (Fig. 3). Since XPS is surface-sensitive, we measured X-ray absorption fine structure (XAFS) in order to obtain information on the bulk of the samples. Along with the results of infrared spectra and elemental analyses, XAFS and XPS are consistently explained supposing that the illumination progressively induced electron transfer (redox reaction) between the $\mathrm{Ag}(\mathrm{I})$ ions and the DM radical anions. The interpretation thus far is consistent with a series of Raman spectra measured continually during the illumination [8], which indicated the formal charge of the DM radical anions continuously changed from -0.5 to $-0.35--0.40$ in accordance with the UV-VIS illumination. Identical specta were obtained several months after ceasing illumination, which means the photochemically induced state as well as the interface between the illuminated and the non-illuminated areas are stable. Powder X-ray diffraction patterns proved that the illuminated samples retained the original (pristine) crystal structure. However, the apparent irreversibility of the phenomena suggests that there should be some difference between the structures before and after illumination. This issue is under investigation.

It is highly possible that one could dope this material simply by illumination with virtually retaining the crystal structure. Thus we tried to make a rectifier from a single crystal of $\operatorname{Ag}(\mathrm{DM})_{2}$ by illuminating on the only half of the long needle crystal. This was expected to remove some conduction electrons from the $\pi$-band of DM columns only in the illuminated part of the crystal, while leaving the rest of the crystal intact. Such partial photochemical 
treatment would force the crystal to take a junction-structure due to the imbalance of the Fermi levels at the interface. After illumination the I-V property curves were measured (Fig. 4). The result clearly shows that electric current exponentially increases when a forward voltage is applied, while it hardly flows when the applied voltage is reversed. Such "one-way" conduction is called rectification, demonstrating its junction-structure and being characteristic to diodes. In other words, the illumination transformed a single crystal into a diode. This method was originally meant for the molecular crystals, yet it could be applicable to thin films and amorphous solids as well as inorganic materials.

\section{References}

[1] P. Batail (Ed.), Chem. Rev. 104 (2004), Thematic Issue for Molecular Conductors.

[2] J.-P. Farges (Ed.), Organic Conductors, Dekker, New York, 1994.

[3] H. S. Nalwa (Ed.), Handbook of Organic Conductive Molecules and Polymers, vol. 1, Wiley, Stuttgart, 1997.

[4] J. M. Williams, J. R. Ferraro, R. J. Thorn, K. D. Carlson, U. Geiser, H. H. Wang, A. M. Kini, M.-H. Whangbo, The Physics and Chemistry of Organic Superconductors (Including Fullerenes), Prentice-Hall, Englewood Cliffs, New Jersey, 1992.

[5] T. Ishiguro, K. Yamaji, G. Saito, Organic Superconductors, 2nd Ed, Springer, New York, 1998.

[6] A. Aumüller, S. Hünig, Liebigs Ann. Chem. (1986) 142. 
[7] T. Naito, T. Inabe, K. Takeda, K. Awaga, T. Akutagawa, T. Hasegawa, T. Nakamura, T. Kakiuchi, H. Sawa, T. Yamamoto, et al. J. Mater. Chem. 11 (2001) 2221.

[8] T. Naito, T. Inabe, H. Niimi, K. Asakura, Adv. Mater. 16 (2004) 1786.

[9] T. Naito, H. Sugawara, T. Inabe, T. Miyamoto, H. Niimi, K. Asakura, Mol. Cryst. Liq. Cryst., in press. 


\section{Figure captions}

Fig. 1. (a) Molecular structure of DM and (b) crystal structure of $\operatorname{Ag}(\mathrm{DM})_{2}$.

Fig. 2. UV-v is illumination effects on the temperature-dependence of the electrical resistivity of an $\mathrm{Ag}(\mathrm{DM})_{2}$ single crystal.

Fig. 3. N1S XPS of $(a)$ pristine, and $(b)$ illuminated samples. Note that a shoulder at lower energy appears more clearly in the illuminated sample, which can be attributed to the increase of the con tribution from formally neutral DM molecules.

Fig. 4. I-V property curve of a single crystal of $\mathrm{Ag}(\mathrm{DM})_{2}$ after UV-VIS illumination on the on ly half of it. The illumination continued for about three weeks from both sides of the needle crystal at room temperature. 


\section{Journal of \\ Non-Crystalline Solids \\ Confirmation of Authorship}

\section{Please save a copy of this file, complete and upload as the "Confirmation of Authorship" file.}

As corresponding author, I Toshio Naito, hereby confirm on behalf of all authors that:

1. This manuscript has not been published, was not, and is not being submitted to any other journal. If presented at a conference, the conference is identified. If published in conference proceedings, substantial justification for re-publication must be presented.

2. All necessary permissions for publication were secured prior to submission of the manuscript.

3. All authors each made a significant contribution to the research reported and have read and approved the submitted manuscript. 
(a)<smiles>CC1=CC(=NC#N)C(C)=CC1=NC#N</smiles>

(b)
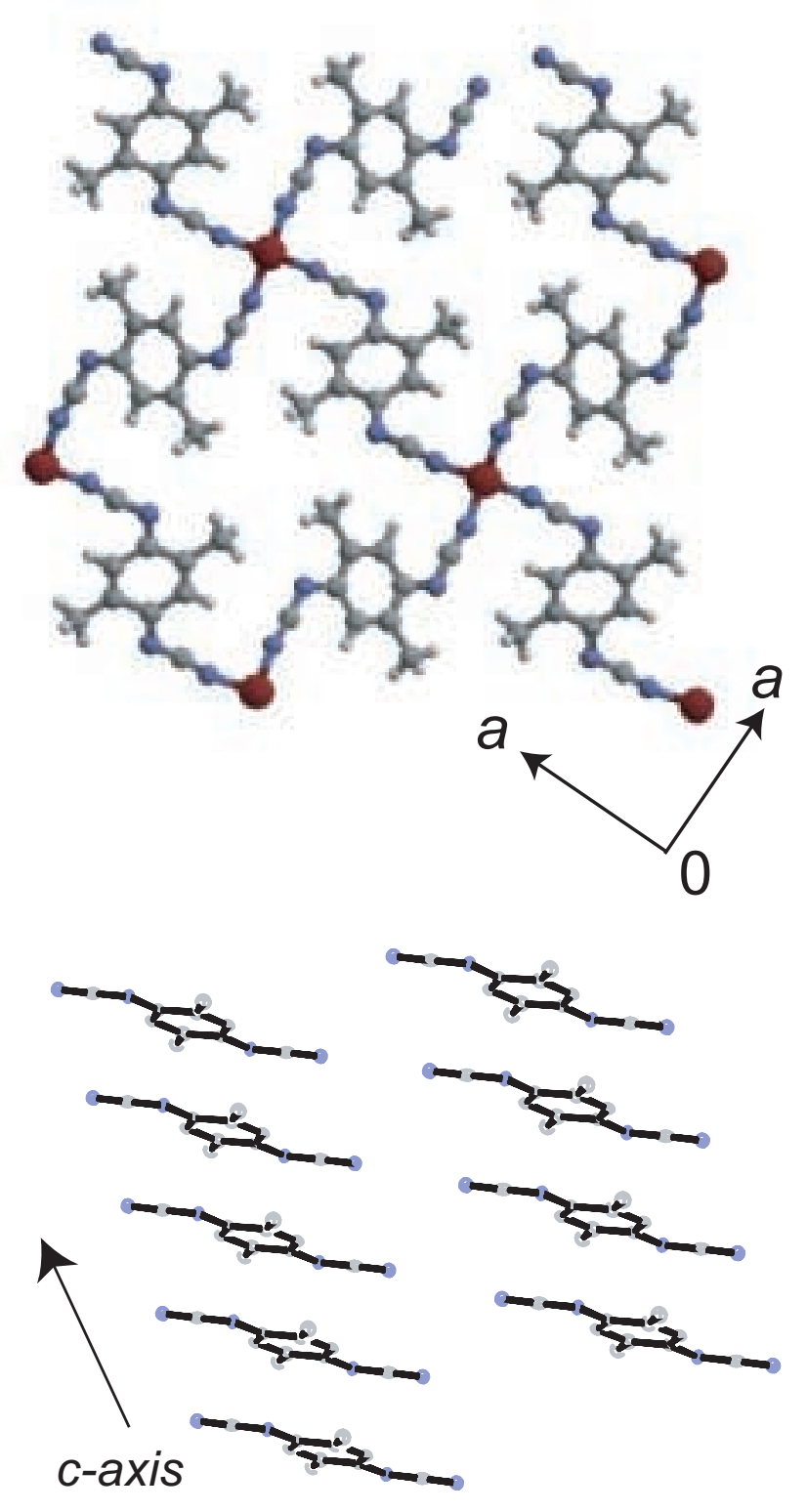

(Fig. 1 T. Naito et al.) 
(Pi)gure 3

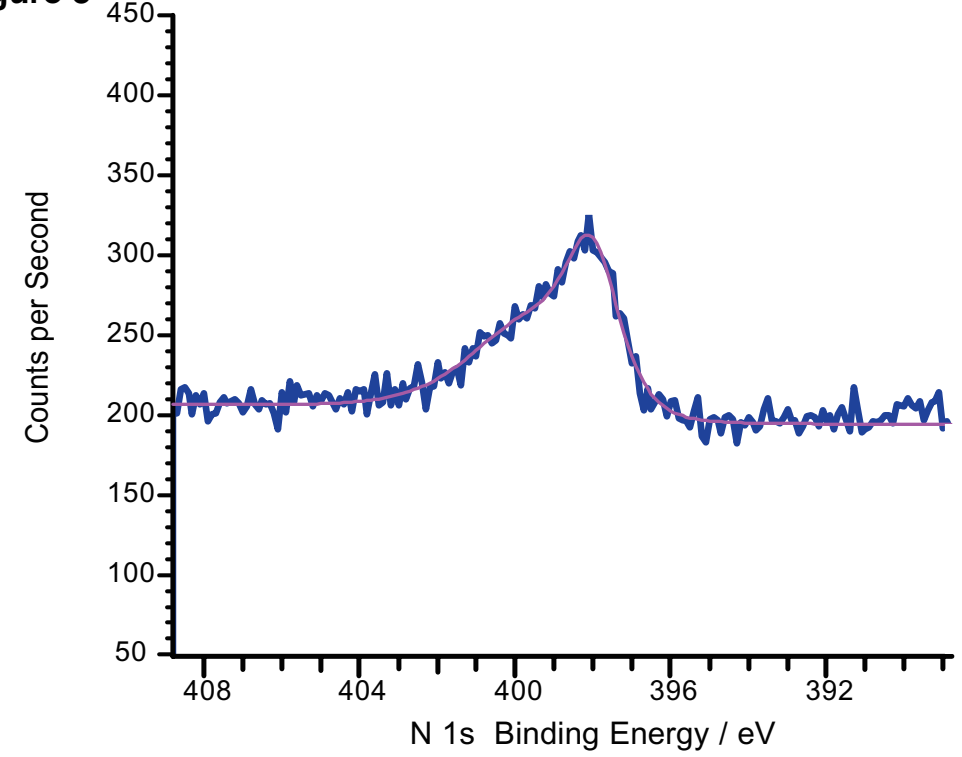

(b)

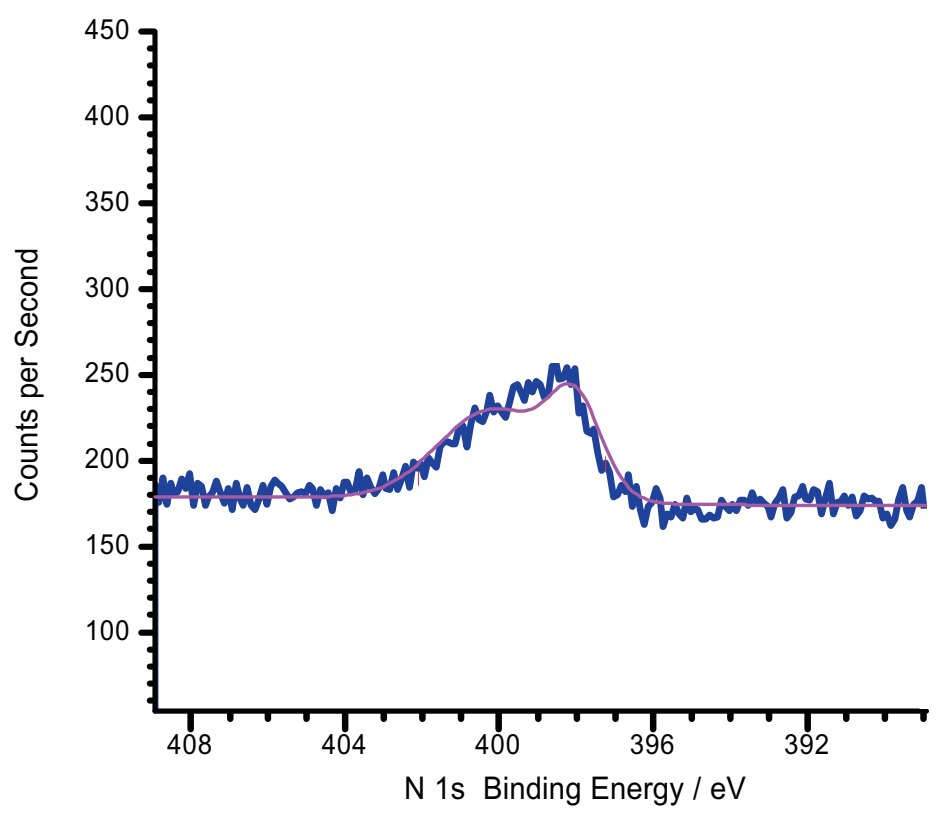

( Fig. 3. T. Naito et al. ) 
